'Facultad de Ciencias Empresariales. Contador

Público y Auditor.

Universidad de Talca.

${ }^{2}$ Unidad de Cuidados

Intensivos. Hospital Base de Curicó.

3Unidad de Cuidados

Intensivos. Hospital Regional de Talca.

${ }^{a}$ Contador Público y Auditor.

Proyecto de investigación diciembre de 2010 hasta el 01 de marzo de 2012,

financiado por el Fondo Nacional de Investigación en Salud (FONIS).

Recibido el 1 de junio de 2012, aceptado el 4 de septiembre de 2012.

Correspondencia a: Sandra Alvear Vega Cel. 97674752

Casilla 321. Talca.

E-mail: salvear@utalca.cl desarrollado entre $01 \mathrm{de}$ Fono 56-71-200337

\section{Costos reales de tratamientos intensivos por paciente y día cama}

\author{
SANDRA ALVEAR ${ }^{1, \mathrm{a}}, \mathrm{JORGE} \mathrm{CANTEROS}^{2}$, \\ JUAN JARA ${ }^{3}$, PATRICIA RODRÍGUEZ $Z^{1, a}$
}

\section{Real daily costs of patients admitted to public intensive care units}

Background: Patient care costs in intensive care units are high and should be considered in medical decision making. Aim: To calculate the real disease related costs for patients admitted to intensive care units of public hospitals. Material and Methods: Using an activity associated costs analysis, the expenses of 716 patients with a mean age of 56 years, mean APACHE score of 20 (56\% males), admitted to intensive care units of two regional public hospitals, were calculated. Patients were classified according to their underlying disease. Results: The costs per day of hospital stay, in Chilean pesos, were \$426,265 for sepsis, \$423,300 for cardiovascular diseases, \$ 418,329 for kidney diseases, \$ 404,873 for trauma, \$398,913 for respiratory diseases, \$379,455 for digestive diseases and \$371,801 for neurologic disease. Human resources and medications determined up to 85 and $12 \%$ of costs, respectively. Patients with sepsis and trauma use 32 and 19\% of intensive care unit resources, respectively. Twenty seven percent of resources are invested in patients that eventually died. Conclusions: A real cost benefit analysis should be performed to optimize resource allocation in intensive care units.

(Rev Med Chile 2013; 141: 202-208).

Key words: Costs and Costs Analysis; Economics; Health Care Costs; Intensive care.

L as prestaciones realizadas en las unidades de cuidados intensivos (UCI) son de alta complejidad e involucran recursos humanos altamente calificados, son dependientes de tecnologías avanzadas y de insumos farmacéuticos de alto costo. En tal sentido, los profesionales de las UCI (s) se enfrentan cotidianamente a tomar decisiones en ambientes con alto nivel de incertidumbre y costos alternativos importantes.

En dicho contexto, los costos también desempeñan un papel importante en el proceso de toma de decisiones y aunque los factores económicos no son el principal criterio en la selección de una alternativa, deben ser parte de la evaluación de los resultados obtenidos en el proceso clínico.

La determinación del costo de cualquier acción depende del propósito o del fin que se ha definido para su determinación. Es así como la información cuantitativa sobre costos, que debe incluirse en una decisión, en el contexto de salud puede variar según la perspectiva en que se realizará el análisis y los objetivos específicos establecidos por cada institución de salud ${ }^{1}$, por ejemplo, Fondo Nacional de Salud (Fonasa), Ministerio de Salud (Minsal), hospitales, servicios clínicos. En síntesis, el costo aun representando uno de los elementos relevantes en los procesos de planificación, control y toma de decisiones, puede dar lugar a diferentes interpretaciones ${ }^{2}$. Por ello es importante precisar y contextualizar su definición.

Se entiende por costo, la suma de erogaciones en que se incurre para la adquisición o producción de un bien o servicio, con la intención de que genere beneficios en el futuro ${ }^{3}$, por ejemplo 
la infraestructura de un hospital, equipamiento, medicamentos, sueldos de los profesionales de la salud, insumos médicos, entre otros.

En cambio el gasto, en tanto este último representa la porción del activo o el desembolso de efectivo que ha contribuido al esfuerzo productivo de un período, que comparado con los ingresos que generó da por resultado la utilidad o pérdida del período ${ }^{4}$. Por ejemplo, los sueldos del personal administrativo del hospital, la suma de los recursos sacrificados en la atención de un paciente egresado de una UCI, entre otros.

Los costos pueden ser clasificados, en primer lugar, según la perspectiva en que se realizará el análisis ${ }^{5}$. Si enfrentamos una perspectiva social, entonces se debiera considerar los costos asociados al cuidado y atención de la salud para todos los miembros de la sociedad, es decir, desde la visión del Estado, hasta el entorno del paciente, incluyendo su familia y trabajo. Paralelamente, si se adopta una perspectiva basada en los pacientes o en la entidad financiadora o aseguradora, solamente se incluirán los costos relacionados con cada grupo en particular, sobre la base del nivel de actividad asociado al objeto de costos. Por ejemplo, costo por patología, por paciente o por día cama, entre otros.

Una vez contextualizado en qué perspectiva se trabajará, los costos son clasificados según el enfoque que se les dé en la toma de decisiones ${ }^{4}$. Las más empleadas en el contexto de la salud chilena son: de acuerdo con su identificación con una actividad (costos directos e indirectos), de acuerdo con su comportamiento (costos fijos y variables), de acuerdo al tiempo en que fueron calculados (costos históricos y predeterminados), de acuerdo con el tipo de sacrificio en que se han incurridos (costos financieros y de oportunidad).

El costo financiero, entendido como los desembolsos monetarios ${ }^{4}$. Es el que afecta directamente a las instituciones de salud, como lo son los hospitales chilenos, en tanto, son base en la toma de decisión y en la evaluación de la gestión de sus directivos. Finalmente, sobre la base de dicho criterio se evalúan los hospitales en su conjunto, es decir, en base a la eficiencia en el uso de los recursos.

Las Unidades de Cuidados Intensivos son centros de producción que concentran procesos clínicos complejos de alto costo financiero, reducido a un número menor de pacientes pero con gran exposición a desenlaces fatales o secuelas invalidantes. Es de amplio interés la identificación de los datos y metodologías para medir y analizar los beneficios y costos de las diferentes prestaciones o contratación de servicios que allí se realizan, teniendo en cuenta las diferencias económicas y socioculturales ${ }^{6}$.

Cada institución hospitalaria debiera disponer de esta información, por ello se planificó un estudio prospectivo que involucre la identificación, medición y valoración de los costos financieros reales incurridos en las Unidades de Cuidados Intensivos de los hospitales públicos en la Región del Maule.

\section{Materiales y Métodos}

La presente investigación ha tenido como objetivo medir los costos financieros reales asociados a las patologías tratadas en las Unidad de Cuidados Intensivos Adulto de dos hospitales públicos de la Región del Maule; Hospital de Curicó y Hospital de Talca. Se trata de un estudio prospectivo que mide los costos por paciente, patologías y días cama de tratamientos intensivos.

La recolección de los datos se ha efectuado entre el 01 de enero y el 31 de diciembre de 2011, utilizando las técnicas de análisis documental, la observación directa no participativa y el análisis de los procesos.

Del total de 716 pacientes incluidos en el estudio, 347 pacientes son del Hospital de Curicó y 369 pacientes son del Hospital de Talca (Tabla 1).

Los pacientes incorporados en el estudio mayoritariamente son hombres (56\%), su edad promedio es de 56 años, registraron un puntaje APACHE promedio de 20 y provienen principalmente del Servicio de Urgencia (37\%). El 97\% de los pacientes tienen como previsión social el Fondo Nacional de Salud (Fonasa) (Tabla 1).

Los pacientes se clasificaron según las siguientes patologías: Sepsis, Cardiovascular, Respiratorias, Neurológicas, Traumas, Digestivas, Renales y Otras, tomando como base el sistema de información definido por la unidades y la diferencia existente entre las prestaciones. En el grupo "Otras" se incluyó post-operatorio electivo, cetoacidosis diabética grave, entre otras.

La Unidad de Cuidados Intensivos de Adultos del Hospital de Curicó, tiene una dotación de 10 
Tabla 1. Características de la población en estudio: Hospital de Talca - Hospital de Curicó

\begin{tabular}{|lcc|}
\hline & $\begin{array}{c}\text { Pacientes } \\
\text { egresados } \\
\text { diciembre de 2011 }\end{array}$ & $\begin{array}{c}\text { Pacientes del } \\
\text { estudio }\end{array}$ \\
\hline Total pacientes & 702 & 716 \\
Distribución por Hospital de Curicó & 340 & 347 \\
Distribución por Hospital de Talca & 362 & 369 \\
\hline Distribución por sexo & $56 \%$ Hombres & $56 \%$ Hombres \\
\hline Edad promedio (media) & 56 años (d.e. 19) & 56 años (d.e. 19) \\
Tipo de previsión & $99 \%$ Fonasa & $97 \%$ Fonasa \\
\hline Puntaje APACHE (media) & 20 & 20 \\
\hline Letalidad & $27 \%$ & $27 \%$ \\
Procedencia: & & \\
Clínicas privadas & $3 \%$ & $3 \%$ \\
Otros hospitales & $16 \%$ & $16 \%$ \\
Otras Unidades del Hospital & $24 \%$ & $25 \%$ \\
Pabellón & $19 \%$ & $20 \%$ \\
Urgencia & $38 \%$ & $37 \%$ \\
\hline
\end{tabular}

Fuente: Elaboración propia.

Tabla 2. Detalle de los costos indirectos

\begin{tabular}{|ll|}
\hline $\mathbf{n}$ & Elemento \\
\hline 01 & Depreciación (equipamiento e inversión a valor de mercado) \\
\hline 03 & Alimentos y bebidas \\
\hline 04 & Coxtiles, vestuario y calzado \\
\hline 05 & Materiales de oficina \\
\hline 06 & Oxígeno y gases clínicos \\
\hline 07 & Otros químicos \\
\hline 08 & Materiales y útiles de aseo \\
\hline 09 & Electricidad \\
\hline 10 & Agua \\
\hline 11 & Gas \\
\hline 12 & Correo \\
\hline 13 & Telefonía fija \\
\hline 14 & Telefonía celular \\
\hline 15 & Acceso a internet \\
\hline 16 & Enlaces de telecomunicaciones \\
\hline 17 & Mantenimiento y reparación de equipos informáticos \\
\hline 18 & Servicios de aseo \\
\hline 19 & Servicios de vigilancia \\
\hline 20 & Pasajes, fletes y bodegajes \\
\hline 21 & Pasaje y traslado paciente \\
\hline 22 & Otras compras de servicios y convenios \\
\hline
\end{tabular}

Fuente: Elaboración propia, a partir de Sistema de Información de Hospitales. camas, cuenta con un equipo de salud conformado por 40 funcionarios: 7 médicos, 13 enfermeras, 13 técnicos paramédicos, 4 auxiliares de servicio, 2 kinesiólogos y una secretaria.

La Unidad de Cuidados Intensivos de Adultos del Hospital de Talca, tiene una dotación de 8 camas, cuenta con un equipo de salud conformado por 45 funcionarios: 8 médicos, 13 enfermeras, 13 técnicos paramédicos, 6 auxiliares de servicio, 4 kinesiólogos y una secretaria.

Se aplicó el Sistema de Costo Basado en Actividades (ABC). Los instrumentos de medición de costos fueron validados el año 2009, en un estudio retrospectivo realizado en la UCI del Hospital de Curicó ${ }^{8}$.

\section{Costos indirectos}

Costos que incluyen el consumo de factores o medios de producción que, por afectar al proceso en su conjunto, no se pueden calcular directamente, sino por distribución ${ }^{4}$. Los elementos que los conforman se muestran en Tabla 2.

1. Identificación, definición y clasificación de actividades: Para nuestro estudio, el término actividad representa a las acciones emprendidas o al trabajo realizado por algún equipo o personas para otras personas.

Con el objetivo de elaborar un diccionario de actividades se entrevistó a cada uno de los grupos de profesionales que conforman el equipo asistencial de una Unidad de Cuidados Intensivos: directivos, médicos, enfermeras, kinesiólogos, paramédicos, auxiliares. A partir de las respuestas obtenidas por cada profesional enfermera, se procedió a su posterior validación, por el grupo de profesionales enfermeras, por la enfermera supervisora y por el médico Jefe de UCI, quienes realizaron un análisis exhaustivo de las 
actividades presentadas. El mismo procedimiento se aplicó para todos los grupos profesionales entrevistados.

2. Una vez identificada y descrita las actividades, la etapa siguiente consistió en determinar el costo de cada actividad. Las actividades consumen recursos, tales como: costo de mano de obra, electricidad, entre otros. Los costos de los recursos identificados se obtuvieron del sistema contable de cada hospital y de las planillas de sueldos.

Para efectos de determinar la tasa de aplicación de los costos indirectos del hospital, asignado a la unidad de cuidados intensivos, se utilizó un Método Proxy ${ }^{9}$. Dicho método, comparado con las Tasas Winsig, permite una aceptable estimación de costos indirectos?.

Para distribuir dichos recursos a las actividades, se consideró el tiempo empleado en cada actividad como unidad de medida de costos, es decir, el tiempo necesario para desarrollar la actividad. Se solicitó a los profesionales entrevistados registrar los tiempos utilizados en cada una de las actividades identificadas, considerando un mes de trabajo y proyectándolo a año de trabajo como horizonte de tiempo.

3. Antes de asignar el costo a los pacientes, fue necesario medir la demanda, que realizaban dichos pacientes de las actividades ${ }^{8}$. Identificamos para cada una de ellas "generadores de actividad", es decir, una medida que nos indicara su frecuencia de realización, expresada en términos de la demanda que ejercen los pacientes sobre las actividades. Como lo son: $\mathrm{N}^{\circ}$ de Hojas de Evolución Médica Cuidados Intensivos, $\mathrm{N}^{\circ}$ de Hojas de Enfermería, entre otros. Dichos "generadores" nos permitió medir el número de veces que se realiza.

4. Finalmente, se asignó el costo desde las actividades a los pacientes. Para ello se determinaron tasas de actividad, dividiendo el costo total de cada actividad por el número total de "generadores de actividad".

\section{Costos directos}

Referido a medios o factores consumidos en el proceso por un producto o servicio, o por un centro de sección de costos sobre lo que se puede calcular prácticamente su medida técnica y económica ${ }^{4}$. Los costos directos identificados en el estudio son: medicamentos, exámenes e insumos.

Se procedió a medir la cantidad de exámenes por pacientes, a partir de los registros de la unidad.
La valoración de dichos exámenes se realizó utilizando el arancel año 2011 del Fondo Nacional de Salud (Fonasa) y no a su costo real.

La cantidad de medicamentos utilizados por paciente, se identificó sobre la base del sistema de registro de la unidad, a partir de las recetas emitidas para cada paciente y su correspondiente aplicación. Los medicamentos se valoraron según el último precio de adquisición registrado por el hospital.

La cantidad de insumos médicos, se identificaron a partir del seguimiento y registro de su consumo en la atención de cada paciente. Entre ellos, se puede destacar: agujas desechables, jeringas, cánula intravenosa, guantes, mascarillas, mariposas, sondas, entre otros. Los insumos de cargo del paciente se valoraron según el último precio de adquisición registrado por el hospital.

\section{Resultados}

Los pacientes incorporados en el estudio mayoritariamente eran hombres, su edad promedio fue de 56 años, registraban un puntaje APACHE promedio de 20, provenían principalmente del Servicio de Urgencia (37\%) y de otras unidades del hospital (25\%). El 97\% de los pacientes tenía como previsión social el Fondo Nacional de Salud (Fonasa) (Tabla 1).

En el estudio realizado no se verificó diferencias significativas por sexo con respecto a los costos por paciente y día cama.

Los pacientes con sepsis, tal como se puede observar en la Tabla 3, consumían la mayor proporción de los recursos aplicados en la unidad (32\%), no obstante, el número de pacientes representa $27 \%$ del total de pacientes en estudio. Con respecto a la misma patología, también se puede observar que del total de costos asociados a ella (32\%), 12\% estaba relacionado con pacientes que finalmente fallecieron.

Los pacientes con patología trauma ocuparon el segundo lugar en la utilización de recursos aplicados en la unidad (19\%), y representan 18\% del total de pacientes del estudio. De dicho porcentaje, $3 \%$ fue utilizado por pacientes fallecidos durante la hospitalización (Tabla 3).

Las patologías que presentaron un mayor costo promedio por día cama fueron: sepsis (\$426.265), cardiovasculares $(\$ 423.300)$, renales $(\$ 418.329)$ 
Costos reales de tratamientos intensivos - S. Alvear et al

Tabla 3. Descripción general de costos por patologías asociadas

\begin{tabular}{|c|c|c|c|c|c|c|c|c|}
\hline & Sepsis & $\begin{array}{c}\text { Cardio- } \\
\text { vasculares }\end{array}$ & Trauma & $\begin{array}{l}\text { Respira- } \\
\text { torias }\end{array}$ & $\begin{array}{l}\text { Neuro- } \\
\text { lógicas }\end{array}$ & Digestivas & Renales & Otros \\
\hline $\mathrm{N}^{\circ}$ pacientes & 194 & 149 & 127 & 73 & 47 & 31 & 18 & 77 \\
\hline Sexo & $\begin{array}{l}54 \% \mathrm{M} \\
46 \% \mathrm{H}\end{array}$ & $\begin{array}{l}37 \% \mathrm{M} \\
63 \% \mathrm{H}\end{array}$ & $\begin{array}{l}27 \% \mathrm{M} \\
73 \% \mathrm{H}\end{array}$ & $\begin{array}{l}36 \% \mathrm{M} \\
64 \% \mathrm{H}\end{array}$ & $\begin{array}{l}40 \% \mathrm{M} \\
60 \% \mathrm{H}\end{array}$ & $\begin{array}{l}65 \% \mathrm{M} \\
35 \% \mathrm{H}\end{array}$ & $\begin{array}{l}44 \% \mathrm{M} \\
56 \% \mathrm{H}\end{array}$ & $\begin{array}{l}70 \% \mathrm{M}, \\
30 \% \mathrm{H}\end{array}$ \\
\hline Fonasa & $95 \%$ & $93 \%$ & $95 \%$ & $94 \%$ & $97 \%$ & $100 \%$ & $100 \%$ & $96 \%$ \\
\hline Letalidad & $40 \%$ & $23 \%$ & $16 \%$ & $30 \%$ & $19 \%$ & $29 \%$ & $33 \%$ & $19 \%$ \\
\hline $\begin{array}{l}\text { Porcentaje total de } \\
\text { costos utilizado }\end{array}$ & $32 \%$ & $15 \%$ & $19 \%$ & $13 \%$ & $8 \%$ & $4 \%$ & $2 \%$ & $7 \%$ \\
\hline $\begin{array}{l}\text { Porcentaje de costos } \\
\text { consumido por } \\
\text { pacientes fallecidos }\end{array}$ & $12 \%$ & $4 \%$ & $3 \%$ & $3 \%$ & $1 \%$ & $1 \%$ & $1 \%$ & $2 \%$ \\
\hline
\end{tabular}

Fuente: Elaboración propia.

Tabla 4. Costos unitarios promedio día cama (pesos, 2011)

\begin{tabular}{|lccccc|}
\hline Patología & $\begin{array}{c}\text { Días cama } \\
\text { promedio }\end{array}$ & $\begin{array}{c}\text { Costo promedio } \\
\text { día cama }\end{array}$ & $\begin{array}{c}\text { Desviación } \\
\text { estándar }\end{array}$ & $\begin{array}{c}\text { Costo promedio } \\
\text { paciente }\end{array}$ & $\begin{array}{c}\text { Desviación } \\
\text { estándar }\end{array}$ \\
\hline Sepsis & 9 & 426.265 & 192.319 & 3.129 .998 & 3.582 .469 \\
\hline Cardiovasculares & 6 & 423.300 & 219.855 & 1.892 .616 & 2.864 .420 \\
\hline Renales & 6 & 418.329 & 167.415 & 2.159 .297 & 1.496 .691 \\
\hline Trauma & 9 & 404.873 & 184.035 & 2.914 .256 & 2.837 .783 \\
\hline Respiratorias & 10 & 398.913 & 152.066 & 3.297 .945 & 2.822 .687 \\
\hline Digestivas & 7 & 379.455 & 227.911 & 2.535 .819 & 3.011 .940 \\
\hline Neurológicas & 13 & 371.801 & 178.165 & 3.403 .509 & 4.450 .806 \\
\hline Otros & 5 & 480.047 & 214.898 & 1.831 .590 & 1.361 .759 \\
\hline
\end{tabular}

Fuente: Elaboración propia.

Tabla 5. Mediana de costos unitarios por día cama (pesos, 2011)

\begin{tabular}{|lccccc|}
\hline Patología & $\begin{array}{c}\text { Días cama } \\
\text { promedio }\end{array}$ & $\begin{array}{c}\text { Mediana costos } \\
\text { día cama }\end{array}$ & $\begin{array}{c}\text { Desviación } \\
\text { cuartílica }\end{array}$ & $\begin{array}{c}\text { Mediana costos } \\
\text { paciente }\end{array}$ & $\begin{array}{c}\text { Desviación } \\
\text { cuartílica }\end{array}$ \\
\hline Renales & 6 & 410.687 & 351.286 & 1.821 .958 & 1.708 .232 \\
\hline Sepsis & 9 & 390.727 & 383.562 & 1.966 .362 & 2.225 .464 \\
\hline Respiratorias & 10 & 373.863 & 380.821 & 2.435 .987 & 2.750 .478 \\
\hline Trauma & 9 & 373.390 & 354.240 & 1.789 .882 & 2.426 .052 \\
\hline Neurológicas & 13 & 348.904 & 346.514 & 1.856 .026 & 2.900 .695 \\
\hline Cardiovasculares & 6 & 346.957 & 389.636 & 1.078 .681 & 1.356 .037 \\
\hline Digestivas & 7 & 287.682 & 112.848 & 1.318 .410 & 475.132 \\
\hline Otros & 5 & 435.596 & 439.396 & 1.346 .193 & 1.652 .996 \\
\hline
\end{tabular}

Fuente: Elaboración propia. 
Costos reales de tratamientos intensivos - S. Alvear et al

Tabla 6. Costos unitarios promedios por pacientes y día cama asociados a las patologías en estudio (pesos)

\begin{tabular}{|c|c|c|c|c|c|c|c|c|c|}
\hline & \multirow{2}{*}{$\begin{array}{c}\text { Costo } \\
\text { promedio } \\
\text { día cama }\end{array}$} & \multicolumn{2}{|c|}{$\begin{array}{c}\text { Costo } \\
\text { actividades }\end{array}$} & \multicolumn{2}{|c|}{$\begin{array}{c}\text { Costo } \\
\text { exámenes }\end{array}$} & \multicolumn{2}{|c|}{$\begin{array}{c}\text { Costo } \\
\text { medicamentos }\end{array}$} & \multicolumn{2}{|c|}{$\begin{array}{l}\text { Costo } \\
\text { insumos }\end{array}$} \\
\hline & & Pesos & $\%$ & Pesos & $\%$ & Pesos & $\%$ & Pesos & $\%$ \\
\hline $\begin{array}{l}\text { Sepsis } \\
(n=194)\end{array}$ & 426.265 & 328.485 & $77 \%$ & 27.923 & $7 \%$ & 46.416 & $11 \%$ & 23.440 & $5 \%$ \\
\hline $\begin{array}{l}\text { Cardiovasculares } \\
(n=149)\end{array}$ & 423.300 & 356.370 & $84 \%$ & 26.957 & $6 \%$ & 15.725 & $4 \%$ & 24.249 & $6 \%$ \\
\hline $\begin{array}{l}\text { Renales } \\
(n=18)\end{array}$ & 418.329 & 345.695 & $83 \%$ & 26.580 & $6 \%$ & 23.138 & $6 \%$ & 22.915 & $5 \%$ \\
\hline $\begin{array}{l}\text { Trauma } \\
(n=127)\end{array}$ & 404.873 & 328.682 & $81 \%$ & 26.472 & $7 \%$ & 28.856 & $7 \%$ & 20.864 & $5 \%$ \\
\hline $\begin{array}{l}\text { Respiratorias } \\
(n=73)\end{array}$ & 398.913 & 327.508 & $82 \%$ & 20.556 & $5 \%$ & 29.181 & $7 \%$ & 21.667 & $5 \%$ \\
\hline $\begin{array}{l}\text { Digestivas } \\
(n=31)\end{array}$ & 379.455 & 277.533 & $73 \%$ & 31.933 & $8 \%$ & 46.582 & $12 \%$ & 23.407 & $6 \%$ \\
\hline $\begin{array}{l}\text { Neurológicas } \\
(n=47)\end{array}$ & 371.801 & 297.371 & $80 \%$ & 22.451 & $6 \%$ & 35.985 & $10 \%$ & 15.994 & $4 \%$ \\
\hline $\begin{array}{l}\text { Otros } \\
(n=77)\end{array}$ & 480.047 & 408.248 & $85 \%$ & 24.428 & $5 \%$ & 26.912 & $6 \%$ & 20.459 & $4 \%$ \\
\hline
\end{tabular}

Fuente: Elaboración propia.

y trauma (\$404.873), con 9, 6, 6 y 9 días cama promedio respectivamente (Tabla 4 ).

Las patologías que presentaron una mayor mediana de costo por día cama fueron: renales $(\$ 410.687)$, sepsis $(\$ 390.727)$, respiratoria $(\$ 373.863)$ y trauma $(\$ 373,390)$, con $6,9,10$ y 9 días cama promedio respectivamente (Tabla 5).

El elemento más representativo en la estructura de costo del día cama fue el costo asociado a las actividades, fluctuó entre $85 \%$ y $73 \%$ por patología. El costo asociado a los profesionales de la salud fue seguido por los medicamentos, que fluctuó entre $12 \%$ y $4 \%$ por patología (Tabla 6 ).

\section{Discusión}

Si bien faltan estudios que caractericen mejor a la población usuaria de cuidados intensivos a nivel nacional, los pacientes incorporados en nuestro estudio son desde el punto de vista epidemiológico una muestra que no difiere significativamente del universo de pacientes que ingresan a las unidades de cuidados intensivos del sector público a nivel nacional, de acuerdo a parámetros de edad, patología y gravedad ${ }^{7,8}$.
Paralelamente, las demandas sanitarias en los servicios de salud y hospitales adscritos a ellos no difieren sustantivamente en este ámbito de prestaciones, tampoco los recursos disponibles para efectuar estas tareas y es relevante tener una aproximación de los costos involucrados en ellas.

El costo asociado a los equipos de salud, que representa el $65 \%$ a nivel global, para dos unidades con una disponibilidad de 8 camas y 10 camas respectivamente, con un índice ocupacional de 91\%, cifra que no se aleja de otros estudios realizados a nivel nacional ${ }^{8-10}$, donde el recurso humano representa $75 \%$ para Unidades de Cuidados Intensivos con 13 camas y con un índice ocupacional de 80\%.

Los principales grupos de patologías analizadas en este estudio expresan los escenarios clínicos en la mayoría de las Unidades de Cuidados Intensivos polivalentes, como son los cuadros cardiovasculares, sepsis, trauma ${ }^{7-10}$.

Los pacientes sépticos y cardiovascular presentan los costos promedios mayores, por patología, $(\$ 426.625 \pm 192.319)$ y $(\$ 423.300 \pm 219.855)$ respectivamente. Es relevante mencionar la cantidad de recursos involucrados en pacientes que finalmente fallecen. 
Costos reales de tratamientos intensivos - S. Alvear et al

A nivel internacional un estudio realizado en Europa, que contempló a 51 unidades de cuidados intensivos, muestra que los pacientes que representan el mayor costo en su tratamiento son los pacientes sépticos, absorbiendo en promedio el $62 \%$ de los recursos, con un costo promedio equivalentes en pesos a $(\$ M 694,6 \pm \$ M 268,9)^{11}$, considerando $\$ 637,27$ por un euro.

A nivel, nacional un estudio retrospectivo de costos de tratamientos intensivos, publicado el año 2010, muestra que los pacientes sépticos demandan la mayor cantidad de recursos, $28 \%$, con un costo promedio de $\$ 270.545^{8}$.

En el presente estudio también se muestra que los motivos de ingresos a las Unidades de Cuidados Intensivos no son homogéneos y que existen niveles de dispersión entre el costo de tratamiento de las distintas patologías; por ejemplo, el día cama promedio de sepsis con respecto al día cama promedio de pacientes neurológicos varía en 15\%. Diferencias que no son consideradas en el arancel fijado por Fonasa.

\section{Conclusión}

Se hace necesario generar instrumentos uniformes de medición de costos, homologarlos y aplicarlos en distintas Unidades de Cuidados Intensivos y publicar sus resultados. La gestión debe permitir procesos de comparación, aprendizaje, y mejoramiento continuo entre las distintas unidades a nivel país.

\section{Limitaciones}

El costo por exámenes es valorado según el arancel Fonasa y no a su costo real. El costo del día cama UCI obtenido en el estudio podría variar frente a una posible sub o sobre valuación del arancel Fonasa.

Presentar en forma agregada los datos de dos UCI(s) de dos hospitales diferentes, no explicita los niveles de eficiencia alcanzados por cada hospital, específicamente el "apalancamiento" de la estructura de costos fijos.

\section{Referencias}

1. Castillo M, Castillo C. La determinación de costos en evaluaciones económicas. En: Anexos Guía Metodoló- gica de Evaluaciones Económicas en Salud.; 2011. p. 63 83. Disponible en: http://desal.minsal.cl/DOCUMENTOS/PDF/2011/Anexos_Guia_Metodologica_EE.pdf. [Consultado el 14 de mayo de 2012].

2. Drummond M, Brien B, Stoddart G, Torrance G. Methods for the Economic Evluation of Health Care Programmes. Tercera edición. New York, USA: Oxford University Press. 2005; 55-95.

3. Horngren $\mathrm{Ch}$, Sundem G, Stratton W. Contabilidad Administrativa. 13a. Edición. México. Pearson Prentice Hall. 2006; 86-97.

4. Mallo C, Kaplan RS, Meljem S, Giménez C. Contabilidad de costos y estratégica de gestión. 1a Edición. Madrid. España. Pearson Prentice Hall. 2000; 33-81.

5. World Health Organization. Making choices in health: WHO guide to cost-effectiveness analysis. (2003). Disponible en: www.who.int/entity/choice/book/en/index. html [Consultado el 14 de mayo de 2012].

6. Seguro social de enfermedad. Financiación sostenible de la salud, cobertura universal y seguro social de enfermedad. 58 a Asamblea Mundial de la Salud. OMS. (2005). Disponible en: www.oms.org. [Consultado el 14 de mayo de 2012].

7. Tomicic V, Espinoza M, Andresen M, Molina J, Calvo $\mathrm{M}$, Ugarte $\mathrm{H}$, et al. Características de los pacientes que reciben ventilación mecánica en unidades de cuidados intensivos: primer estudio multicéntrico chileno. Rev Med Chile 2008; 136 (8): 959-67. Disponible en www. scielo.cl. [Consultado el 14 de mayo de 2012].

8. Alvear S, Cantero J, Rodríguez P. Estudio retrospectivo de costos de tratamientos intensivos por paciente y día cama. Rev Med Chile 2010; 138 (5): 558-66. Disponible en www.scielo.cl. [Consultado el 14 de mayo de 2012].

9. MINSAL. 2010. Informe Final. Estudio de Costo - Efectividad de Intervenciones en Salud. Ministerio de Salud. Informe Final. Disponible en: http://desal.minsal.cl/ DOCUMENTOS/PDF/GES/1.2/01CostoEfectividad.pdf [Consultado el 14 de mayo de 2012].

10. Dougnac A, Mercado L, Cornejo R, Cariaga M, Hernández G, Andresen M, et al. Prevalencia de sepsis grave en las Unidades de Cuidados Intensivo. Primer estudio nacional multicéntrico. Rev Med Chile 2007; 135 (5): 620-630. Disponible en www.scielo.cl. [Consultado el 14 de mayo de 2012].

11. Moerer O, Plock E, Mgbor U, Schmid A, Schneider H, Wischnewsky $\mathrm{M}$, et al. A German national prevalence study on the cost of intensive care: an evaluation from 51 intensive care units. Critical Care 2007; 11 (3): R69. Disponible en: http://ccforum.com. [Consultado el 14 de mayo de 2012]. 